\title{
Prolonged Bradycardia Induced by Bevacizumab-Associated Infusion Reaction in Advanced Metastatic Colon Cancer
}

\author{
Yuichi Moria, c, Shin-ei Kudo ${ }^{\text {a }}$, Kunihiko Wakamura ${ }^{a}$, Shingo Matsudaira ${ }^{a}$, Masashi Misawa ${ }^{a}$, \\ Yuusaku Sugihara $^{\mathrm{b}}$, Hideyuki Miyachi ${ }^{\mathrm{a}}$, Atsushi Katagiria ${ }^{\mathrm{a}}$, Toshiyuki Baba ${ }^{\mathrm{a}}$
}

\begin{abstract}
A 71-year-old man complaining of bilateral leg edema and general fatigue was admitted to the hospital and diagnosed as advanced sigmoid colon cancer with multiple liver and lung metastasis. The 5-FU/leucovorin/oxaliplatin (FOLFOX)/bevacizumab was started as a first-line therapy. In the second course of FOLFOX/bevacizumab, just after finishing infusion of bevacizumab, he felt severe thrill with chill and got fever (maximum of $38.1{ }^{\circ} \mathrm{C}$ ). Since the symptom was diagnosed as an infusion reaction induced by bevacizumab, treatment with steroid and histamine antagonist was performed. His symptom disappeared soon after the treatment; however, his heart rate came lower below 50/minute 5 hours after the infusion reaction (his heart rate was routinely around 60/minute). The 12-lead electrocardiogram showed sinus bradycardia. The bradycardia (30 - 49/minute) continues at least 60 hours and spontaneously recovered. Literature search of PubMed showed just two papers implying the relation between bevacizumab and bradycardia. This case suggested that bradycardia induced by bevacizumab-associated infusion reaction should be carefully followed up and might be observed with admission if necessary.
\end{abstract}

Keywords: Infusion reaction; Colon cancer; Bevacizumab; Bradycardia

\section{Introduction}

Bevacizumab in combination with fluorouracil/folinic acid/ oxaliplatin (FOLFOX) is a strong option as first-line chemotherapy for metastatic colorectal cancer [1]. Bevacizumab is a humanized recombinant monoclonal antibody which binds to

Manuscript accepted for publication January 29, 2015

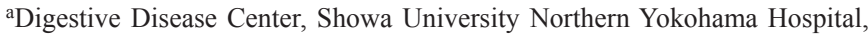
Yokohama, Japan

bepartment of Gastroenterology and Hepatology, Okayama University Graduate School of Medicine and Dentistry, Okayama, Japan

${ }^{\mathrm{c} C}$ Corresponding Author: Yuichi Mori, Digestive Disease Center, Showa University Northern Yokohama Hospital, 35-1, Chigasakityuo, Tsuzuki-ku, Yokohama, Japan. Email: ibusiginjp@hotmail.com

doi: http://dx.doi.org/10.14740/jmc2066w and blocks the activity of all isoforms of vascular endothelial growth factor-A. Monoclonal antibodies, including rituximab, alemtuzumab, trastuzumab, bevacizumab, cetuximab and panitumumab, have improved the treatment of various malignancies. Although generally better tolerated with less toxicity than conventional anticancer agents, monoclonal antibodies may cause infusion-related reactions like other infusional agents. The incidence of infusion reactions varies by agent, but severe events occur only occasionally, mostly with the first or second infusion. Although the exact etiology of infusion reactions remains unclear, they may arise via either IgE- or non-IgEdependent mechanisms [2]. Infusion reaction shows various symptoms: allergic reaction, arthralgia, bronchospasm, cough, dizziness, drug fever, dyspnea, fatigue, headache, hypotension/hypertension, nausea, pruritus, rash, tachycardia and bradycardia. However, bradycardia induced by bevacizumabassociated infusion reaction is a rare event, and its mechanism and precise incidence is still unknown. Here, we present a case of prolonged bradycardia induced by bevacizumab-associated infusion reaction in advanced metastatic colon cancer.

\section{Case Report}

A 71-year-old man complaining of bilateral leg edema and general fatigue for 4 months visited the Showa University Northern Yokohama Hospital. He has no past history and allergic history. Laboratory examination revealed mild anemia, low albumin, mild liver dysfunction and high level tumor marker (Table 1). Computed tomography (CT) was done to clarify the cause of the edema, which revealed that the patient was suffering from multiple liver and lung metastatic tumors. Thus, he underwent colonoscopy in order to detect the origin of the metastasis, which revealed advanced cancer in the sigmoid colon. Biopsy specimens showed tubular adenocarcinoma with K-ras mutation of G12S. Therefore, he was diagnosed as advanced sigmoid colon cancer with multiple liver and lung metastasis. After obtaining patient's informed consent, 5-FU/leucovorin/ oxaliplatin (FOLFOX4)/bevacizumab was started as a firstline therapy with admission. Operation was not performed prior to the chemotherapy, because there was not severe stenosis due to the colon cancer.

After being made of a central vein port, he underwent the first course of $80 \%$ dosed FOLFOX4/bevacizumab. He expe- 
Table 1. Laboratory Findings on the Previous Day of the Infusion Reaction

\begin{tabular}{ll}
\hline WBC & $4,790 / \mu \mathrm{L}$ \\
$\mathrm{Hb}$ & $10.2 \mathrm{~g} / \mathrm{dL}$ \\
$\mathrm{Plt}$ & $26.9 \times 10^{4} / \mu \mathrm{L}$ \\
$\mathrm{PT}$ & $66.70 \%$ \\
APTT & $29.4 \mathrm{~s}$ \\
$\mathrm{D}-\mathrm{dimer}$ & $10.6 \mu \mathrm{g} / \mathrm{mL}$ \\
$\mathrm{TP}$ & $5.1 \mathrm{~g} / \mathrm{dL}$ \\
$\mathrm{UA}$ & $2.4 \mathrm{mg} / \mathrm{dL}$ \\
$\mathrm{BUN}$ & $14.5 \mathrm{mg} / \mathrm{dL}$ \\
$\mathrm{Cre}$ & $0.66 \mathrm{mg} / \mathrm{dL}$ \\
$\mathrm{TB}$ & $0.5 \mathrm{mg} / \mathrm{dL}$ \\
$\mathrm{AST}$ & $43 \mathrm{U} / \mathrm{L}$ \\
$\mathrm{ALT}$ & $20 \mathrm{U} / \mathrm{L}$ \\
$\gamma-\mathrm{GTP}$ & $66 \mathrm{U} / \mathrm{L}$ \\
$\mathrm{CK}$ & $76 \mathrm{U} / \mathrm{L}$ \\
$\mathrm{ALP}$ & $605 \mathrm{U} / \mathrm{L}$ \\
$\mathrm{LDH}$ & $488 \mathrm{U} / \mathrm{L}$ \\
$\mathrm{Na}$ & $137 \mathrm{mEq} / \mathrm{L}$ \\
$\mathrm{Cl}$ & $106 \mathrm{mEq} / \mathrm{L}$ \\
$\mathrm{K}$ & $4.5 \mathrm{mEq} / \mathrm{L}$ \\
$\mathrm{Ca}$ & $7.8 \mathrm{mEq} / \mathrm{L}$ \\
$\mathrm{CRP}$ & $4.34 \mathrm{mg} / \mathrm{dL}$ \\
$\mathrm{CEA}$ & $1,249 \mathrm{ng} / \mathrm{mL}$ (data upon admission) \\
$\mathrm{CA} 19-9$ & $5,310 \mathrm{U} / \mathrm{mL}$ (data upon admission) \\
\hline & \\
\hline
\end{tabular}

rienced grade- 3 anorexia during this course, but spontaneously recovered. Three weeks after the first infusion of FOLFOX4/ bevacizumab, he underwent the second course of FOLFOX4/ bevacizumab with $60 \%$ dosed FOLFOX4. During this course, just after finishing infusion of bevacizumab, he felt severe thrill with chill and got fever of $38.1{ }^{\circ} \mathrm{C}$. Since the symptom was diagnosed as an infusion reaction induced by bevacizumab, the chemotherapy was immediately ceased and treatment with steroid, and histamine antagonist (200 mg hydrocortisone sodium succinate, $5 \mathrm{mg}$ d-chlorpheniramine maleate and $20 \mathrm{mg}$ famotidine) was performed. His symptom disappeared soon after the treatment, and FOLFOX4 was resumed from the next day morning. However, his heart rate came lower below 50/ $\min 5 \mathrm{~h}$ after the infusion reaction (his heart rate was routinely around 60/min (Fig. 1)). Severe bradycardia ( $<40 / \mathrm{min})$ continues at least $9 \mathrm{~h}$ on the next day of the infusion reaction (Fig. 2 ). There was no abnormality of electrolyte data on the previous day of the infusion reaction (Table 1). The bradycardia did not accompany blood pressure reduction or any symptom such as loss of consciousness. Figure 3 shows the course of the treatment of the second course FOLFOX4 plus bevacizumab.

The third course of FOLFOX4/bevacizumab was started 2 weeks after the second course. From the third course, bevacizumab was infused in 90 min with premedication $(13.2 \mathrm{mg}$ dexamethasone, $5 \mathrm{mg}$ d-chlorpheniramine maleate and $20 \mathrm{mg}$ famotidine) in order to prevent the recurrence of the infusion reaction. Total of 18 courses of FOLFOX4/bevacizumab were able to be performed without any infusion reaction until his disease was diagnosed as progressive disease with CT.

Cardiac function was evaluated 3 months after the infusion reaction in order to assess the effect of the previous severe bradycardia. The 12-lead electrocardiogram revealed mild bradycardia (48/min) with first degree atrium-venticle block. Holter-electrocardiogram also revealed first degree atrium-

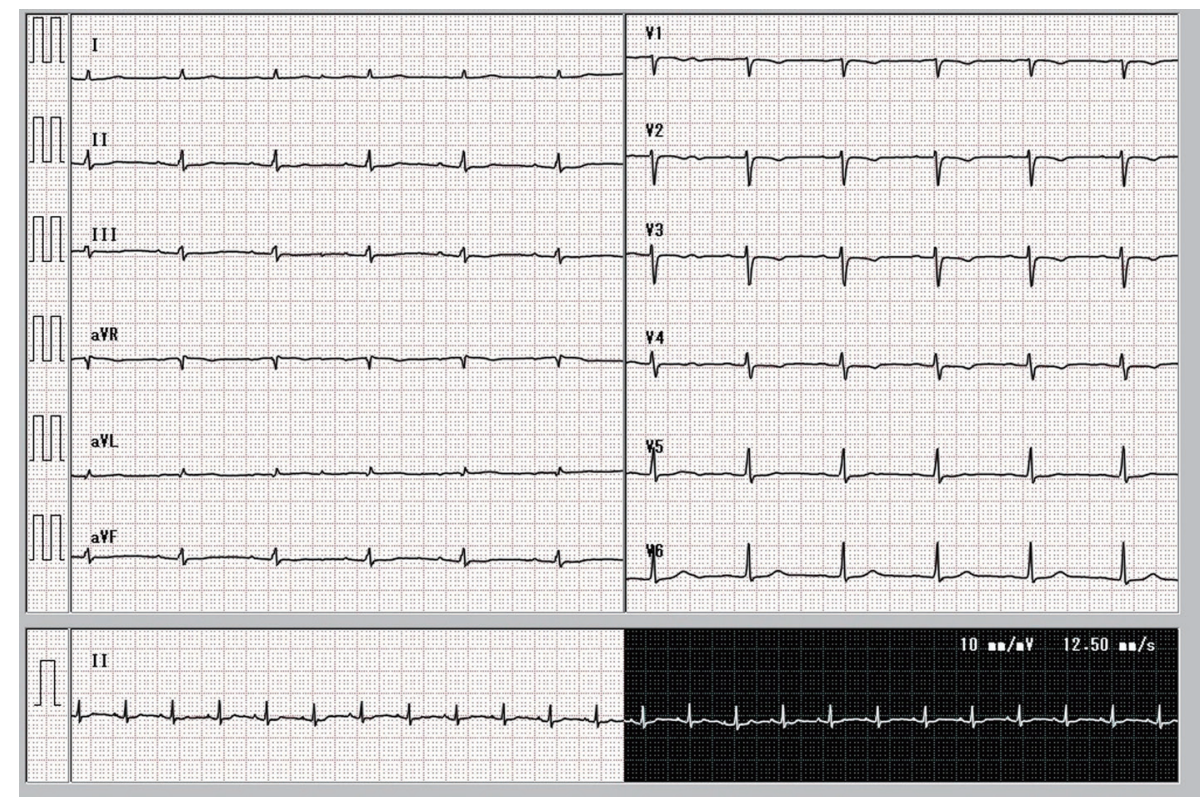

Figure 1. Electrocardiography before the chemotherapy (1 month before the chemotherapy). Heart rate was $70 /$ min, sinus rhythm. 


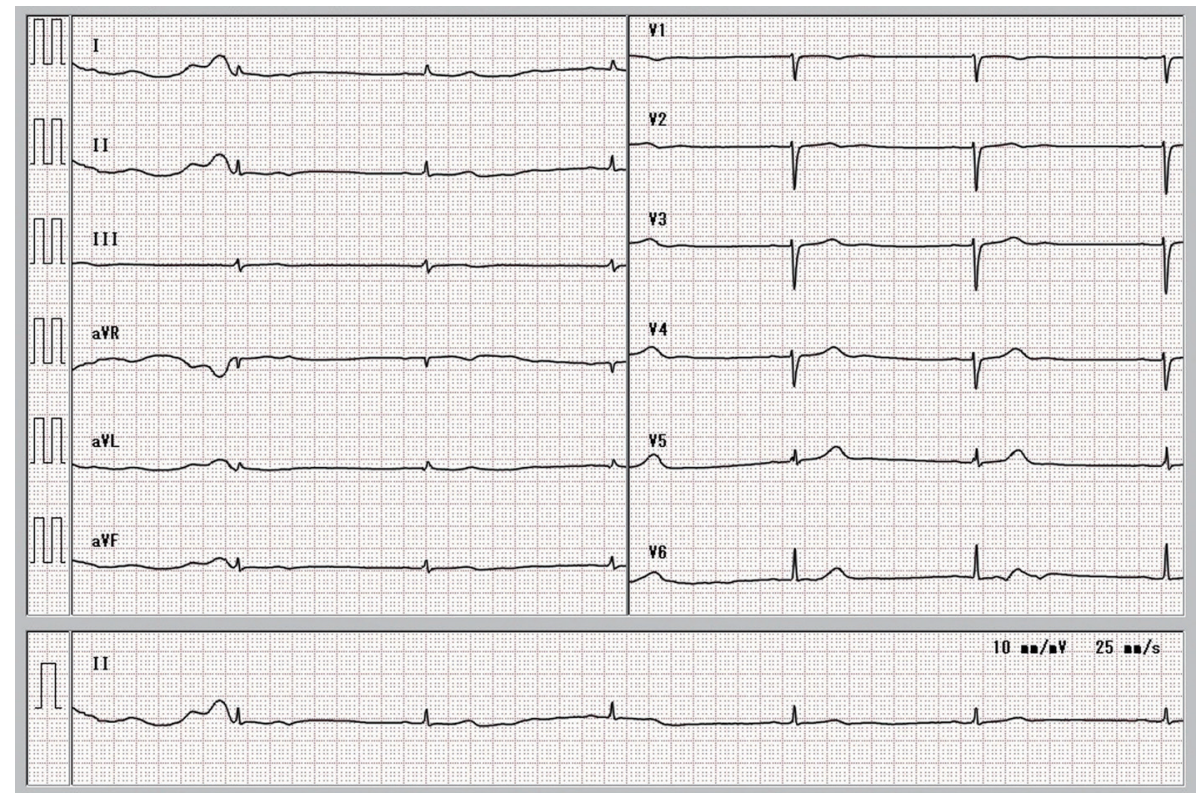

Figure 2. Electrocardiography on 1 day after the infusion reaction. Heart rate was $36 / \mathrm{min}$, sinus rhythm.

venticle block with heart rate of between 40/min and 81/min. The echocardiography revealed normal ejection fraction of $64 \%$ and mild aortic regurgitation with normal wall motion.

\section{Discussion}

Infusion reactions are the generic term for the acute characteristic harmful reactions commonly associated with monoclonal antibody treatment $[2,3]$. Infusion reaction induced by bevacizumab is relatively rare. It was reported to occur in $<3 \%$ of patients during the first infusion of bevacizumab [3]. Focus- ing on the symptom of bradycardia, the incidence was very low; $0.13 \%(4 / 3,109)$ of the patient experienced bradycardia induced by bevacizumab in Japan according to the interview form of bevacizumab in Japan [4].

In this case, mild bradycardia with first degree atriumventicle block was observed even 3 months after the infusion reaction. Only one paper implied the relation between bevacizumab and bradycardia in PubMed [5], but the mechanism of the bradycardia induced by the infusion reaction and the reason why the bradycardia prolonged were still unknown. The exact mechanism responsible for infusion reactions to monoclonal antibodies is still not known. Monoclonal antibodies may in-

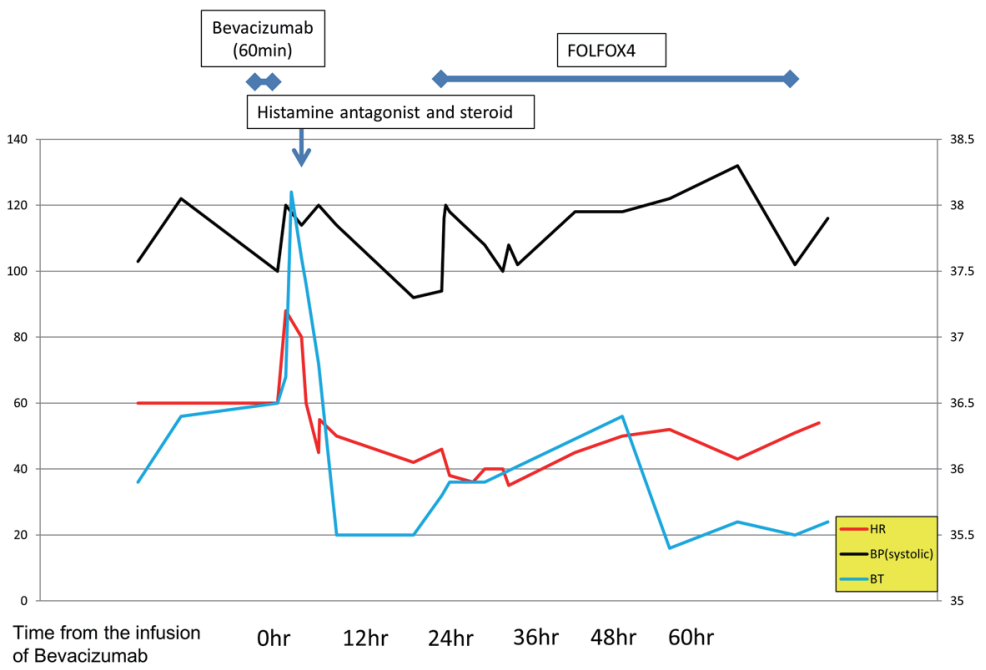

Figure 3. The course of the treatment of the second course FOLFOX4 plus bevacizumab. 
teract with their molecular targets on circulating blood cells, tumor cells or effector cells recruited to the tumor site, thereby promoting the release of inflammatory cytokines, which can produce a wide range of symptoms such as bradycardia $[2,3]$.

In summary, bradycardia induced by bevacizumab-associated infusion reaction is a rare event, but should be carefully followed up and observed with admission if necessary.

\section{Conflict of Interest}

The authors declare that they have no conflict of interest.

\section{References}

1. Saltz LB, Clarke S, Diaz-Rubio E, Scheithauer W, Figer
A, Wong R, Koski S, et al. Bevacizumab in combination with oxaliplatin-based chemotherapy as first-line therapy in metastatic colorectal cancer: a randomized phase III study. J Clin Oncol. 2008;26(12):2013-2019.

2. Chung $\mathrm{CH}$. Managing premedications and the risk for reactions to infusional monoclonal antibody therapy. Oncologist. 2008;13(6):725-732.

3. Lenz HJ. Management and preparedness for infusion and hypersensitivity reactions. Oncologist. 2007;12(5):601609.

4. Interview form of Bevacizumab in Japan (written in Japanese). 2013.

5. Zekri JM. Case report and review of literature: temporary asymptomatic sinus bradycardia with carboplatin, paclitaxel and bevacizumab: under-reported in clinical trials and under-disclosed in practice. Gulf $\mathrm{J}$ Oncolog. 2011;(10):60-64. 